\title{
Testing of Droplet-Based Microelectrofluidic Systems
}

\author{
Fei Su, Sule Ozev, and Krishnendu Chakrabarty \\ Department of Electrical \& Computer Engineering \\ Duke University, Durham, NC 27708 \\ E-mail: \{fs, sule, krish $\} @$ ee.duke.edu
}

\begin{abstract}
Composite microsystems that integrate mechanical and fluidic components are fast emerging as the next generation of system-on-chip designs. As these systems become widespread in safety-critical biomedical applications, dependability emerges as a critical performance parameter. In this paper, we present a costeffective concurrent test methodology for droplet-based microelectrofluidic systems. We present a classification of catastrophic and parametric faults in such systems and show how faults can be detected by electrostatically controlling and tracking droplet motion. We then present tolerance analysis based on Monte-Carlo simulations to characterize the impact of parameter variations on system performance. To the best of our knowledge, this constitutes the first attempt to define a fault model and to develop a test methodology for droplet-based microelectrofluidic systems.
\end{abstract}

\section{Introduction}

Composite microsystems that incorporate microelectromechanical (MEMS) and microelectrofluidic systems (MEFS) are fast emerging as the next generation of system-on-chip designs [1, 2, 3]. These systems combine microstructures with solid-state electronics to integrate electrical, mechanical, and fluidic energy domains. The combination of microelectronics and microstructure is enabling a new class of integrated systems targeted at enviornmental sensing, actuation and control, biomedial analysis, agent detection, and precision fluid dispensing $[4,5,6]$.

MEFS research addresses the miniaturization of composite devices and systems, and the study of new applications associated with the handling of liquids and gases. MEFS not only offer size reduction, e.g., in small medical implants [7] and minimal-invasive surgery [8], but they also reduce power consumption. MEFS also allow us to control small amounts of fluids for precision dispensing (micro-dosing) [9] and reduce reagent consumption for on-line chemical analysis and real-time process monitoring. By scaling down the concentration of chemical samples, simpler sensing techniques can be utilized to replace costly practices involving batch analysis, sample pre-treatment, and frequent calibration.
Moreover, small sample volumes reduce storage cost, enhance uninterrupted use, and benefit a number of medical procedures.

The 2001 International Technology Roadmap for Semiconductors (ITRS) [10] clearly identifies the integration of electro-chemical and electro-biological MEFS as one of the system-level design challenges that will be faced beyond 2007, when feature sizes shrink below $65 \mathrm{~nm}$. The use of MEFS for biomedical applications, such as micro-total analysis systems ( $\mu \mathrm{TAS}$ ), chem/bio sensors, and lab-on-a-chip has received considerable attention recently $[3,11]$. As MEFS become widespread in chem/bio sensors and safety-critical biomedical applications, system dependability emerges as an essential performance parameter. In order to ensure dependability, composite microsystems incorporating MEFS components must be adequately tested. Therefore, there is a pressing need for efficient testing methodologies for MEFS-based sensors and actuators. In fact, the ITRS 2001 document recognizes the need for new test methods for disruptive device technologies that underly MEMS and sensors, and highlights it as one of the five difficult test challenges beyond 2007.

Over the past decades, the focus in testing research has broadened from logic and memory test to include the testing of analog and mixed-signal circuits. More recently, MEMS testing has also received attention, especially fault modeling and fault simulation in surface micromachined MEMS [12, 13]. The testing of composite microsystems containing fluidic components has however received much less attention. The only reported work to date in this area is limited to the testing of continuous-flow MEFS [14, 15, 16]. However, continuous-flow systems, which are based on the use of micro-pumps, micro-valves, and micro-channels, are cumbersome and less amenable to miniaturization. Recently flow-FET based MEFS have been proposed; however, their flow rates are limited to only $50 \mu \mathrm{m} / \mathrm{s}$ [16]. As a result, electrostatically-actuated, droplet-flow MEFS have recently been proposed as an alternative to continuous-flow systems. Such droplet-flow systems, which we review in Section 2, offer exciting possibilities in the realm of massively-parallel DNA analysis, automated drug synthesis, and real-time molecular detection and recognition. Advances in test technology are now required to facilitate the continued 


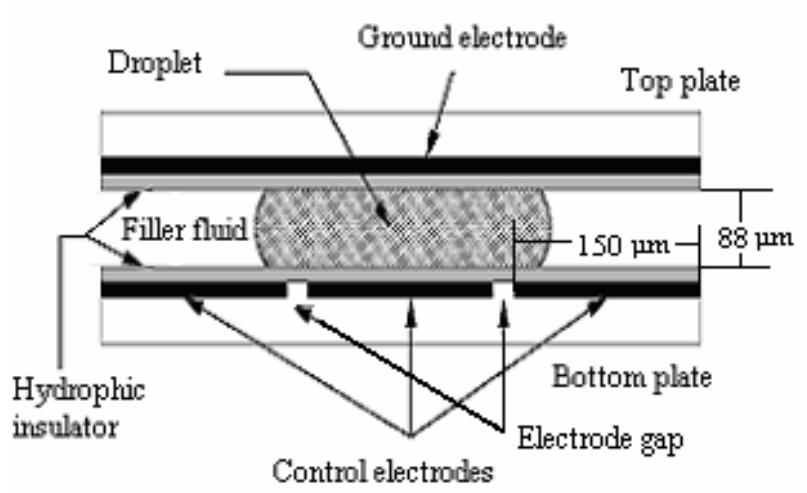

Figure 1. Actuation mechanism for droplet motion.

growth of composite microsystems based on droplet flow.

In this paper, we focus on cost-effective concurrent test methodologies for droplet-based MEFS. We present a list of likely defects in such systems and classify them as catastrophic and parametric faults. We present a novel fault detection mechanism for both catastrophic and parametric faults. Fault detection is based on tracking droplet movement through the unused portions of the system. It can be implemented without disrupting the normal mode of operation, and with negligible hardware overhead. Finally, we present tolerance analysis based on Monte-Carlo simulation to characterize the impact of variations in physical and fluidic parameters on the system performance. To the best of our knowledge, this is the first attempt to define a fault model and develop a test methodology for droplet-based MEFS.

The organization of the remainder of the paper is as follows. In Section 2, we present an overview of electrowetting-actuated droplet-based microelectrofluidic systems. Section 3 presents a list of possible defects and the testing scheme for catastrophic faults. Section 4 discusses parametric fault testing and its evaluation in terms of minimum detectable parameter deviations. Conclusions are drawn in Section 5.

\section{Electrowetting-Actuated Droplet- Based Microelectrofluidic Systems}

Electrowetting-based actuation of microelectrofluids has recently been proposed for optical switching [17], chemical analysis [18], and rotating yaw rate sensing [19]. By varying the electrical potential along a linear array of electrodes, electrowetting can be used to move nanoliter volume liquid droplets along this line of electrodes [18]. Droplets can also be transported, in user-defined patterns and under clocked-voltage control, over a twodimensional array of electrodes without the need for pumps and valves. The basic component of a dropletbased MEFS is shown in Figure 1. The droplet, usually containing biomedical samples, and the filler medium, such as silicone oil, are sandwiched between two parallel

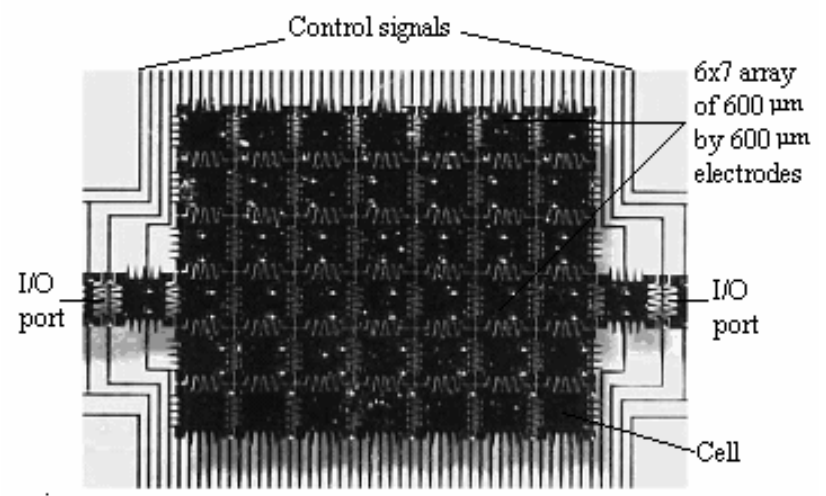

Figure 2. A two-dimensional electrode array for microfluidics.

glass plates. The bottom plate contains a patterned array of individually controllable electrodes, while the top plate is coated with a ground electrode. The hydrophobic dielectric insulator is added to the top and bottom plates to decrease the wettability of the surface and to add capacitance between droplet and control electrode.

The basic principle of microdroplet transportation is to electrostatically control the interfacial tension at the droplet/insulator interface. A control voltage is applied to an electrode adjacent to the droplet and at the same time the electrode just under the droplet is deactivated. This causes an accumulation of charge in the droplet/insulator interface, resulting in a surface tension gradient across the gap between the adjacent electrodes, which consequently causes the transportation of the droplet. The velocity of the droplet can be controlled by adjusting the control voltage $(0 \sim 90 \mathrm{~V})$, and droplets can be moved at speeds of up to $20 \mathrm{~cm} / \mathrm{s}$. Based on this principle, microfluidic droplets can be moved freely to any location of a twodimensional array; see Fig. 2 [22]. This design is ideally suited for a large-scale integrated microfluidic system, which is expected to be extensively used for various biomedical applications, such as DNA sequencing and biomolecule detection, in the near future. The electrodes can be viewed as building blocks in nanotechnology such that huge networks of nano devices can be easily assembled. Each electrode set can be viewed as a pump at the nano/pico-scale, and an array of these pumps can be used for biochemical assays.

Fluid droplets are introduced to the device from the input-output (I/O) ports on the boundary of an array. Droplets in the array have identical volumes. Hence, this device is called a unit-flow device. It is desirable to maintain the unit-flow constraint since the rate of chemical and biomedical reaction grows exponentially with the growth of droplet volume [18]. In a unit flow enviroment, the routes that droplets travel and their rendezvous points are programmed into a micro-controller that controls the voltages of the electrodes. 


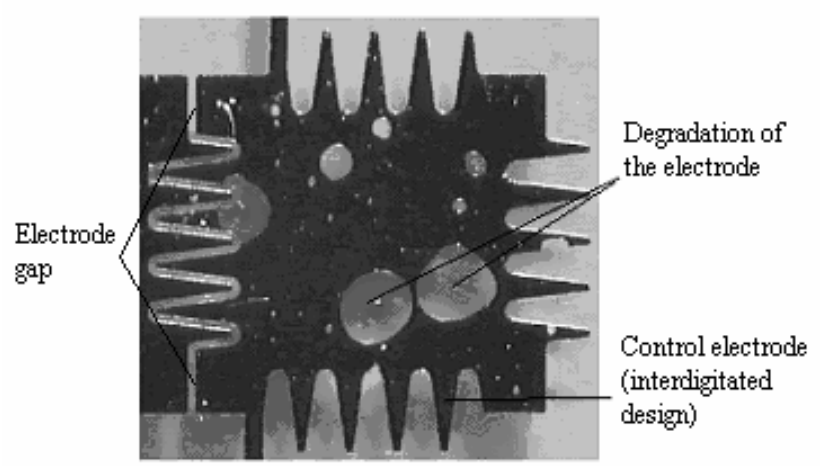

Figure 3. Top-level view of a cell: impact of insulator degradation.

\section{Classification of Faults in Droplet- Based MEFS}

It is evident that droplet-based microelectrofluidic systems exhibit behavior resembling analog and mixedsignal devices. Therefore, we classify the faults in these systems as being either catastrophic or parametric, along the line of fault classification for analog circuits [21]. Catastrophic (hard) faults lead to a complete malfunction of the system, while parametric (soft) faults cause a deviation in the system performance. A parametric fault is detectable only if this deviation exceeds the tolerance in system performance. Due to their underlying mixed technology and multiple energy domains, microelectrofluidic systems exhibit failure mechanisms and defects that are significantly different from the failure modes in analog integrated circuits.

Catastrophic faults in MEFS may be caused by the following physical defects:

- Dielectric breakdown: The breakdown of the dielectric at high voltage levels creates a short between the droplet and the electrode. When this happens, no charge can be stored in the interface. The insulator is damaged by excessive current so that a short subsequently exists even at lower voltages. The feasibility of electrowetting depends on the amount of energy stored in the capacitor formed between the droplet and electrode. If the capacitor is shorted out, there is no charge storage and hence no electrowetting can occur. This catastrophic fault eventually prevents droplet transportation.

- Short between the adjacent electrodes: If a short occurs between two adjacent electrodes, the two electrodes shorted effectively form one longer electrode. When a droplet resides on this electrode, it is no longer large enough to overlap the gap between adjacent electrodes. As a result, the actuation of the droplet can no longer be achieved.

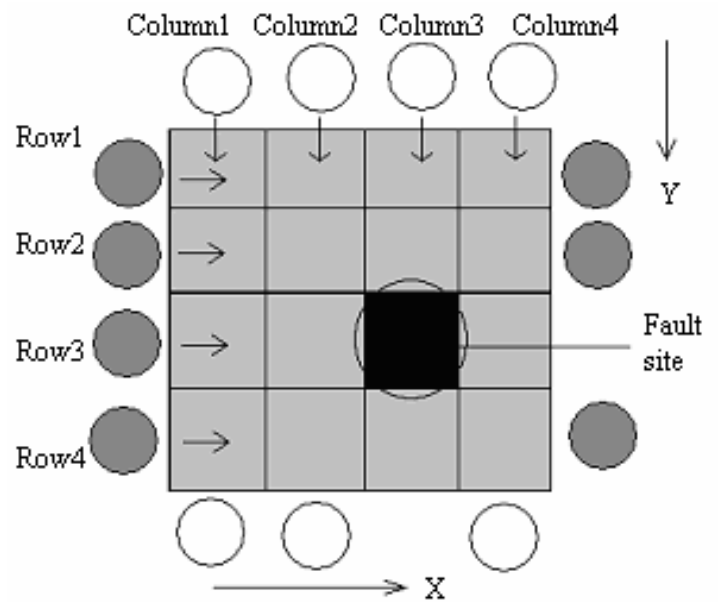

Figure 4. Illustration of the catastrophic fault testing scheme.

- Degradation of the insulator: This degradation effect is unpredictable and may become apparent gradually during the operation of the microfluidic system. Figure 3 illustrates the electrode degradation due to insulator degradation defect [22]. A consequence of insulator degradation is that droplets often fragment and their motion is prevented because of the unwanted variation of surface tension forces along their flow path.

- Open in the metal connection between the electrode and the control source: This defect results in a failure of charging electrode while trying to drive the droplet.

Physical defects that cause parametric faults include the following:

- Geometrical parameter deviation: The deviation in insulator thickness, electrode length and height between parallel plates may exceed their tolerance value.

- Change in viscosity of droplet and filler medium. These deviations can occur during the operation due to an unexpected biochemical reaction, or changes in operational environment, e.g., temperature variation.

Faults in microelectrofluidic systems can also be classified based on the time at which they appear. Therefore, system failure or degraded performance can either be caused by manufacturing defects or they might be due to parametric variations. Testing of manufacturing defects, such as a short between the adjacent electrodes or a deviation in the value of the geometrical parameters, should be performed immediately after production. However, operational faults, such as degradation of the insulator or change in fluid viscosity, can occur throughout the lifetime of the system. Therefore, concurrent testing during system operation is necesssary for such faults. 


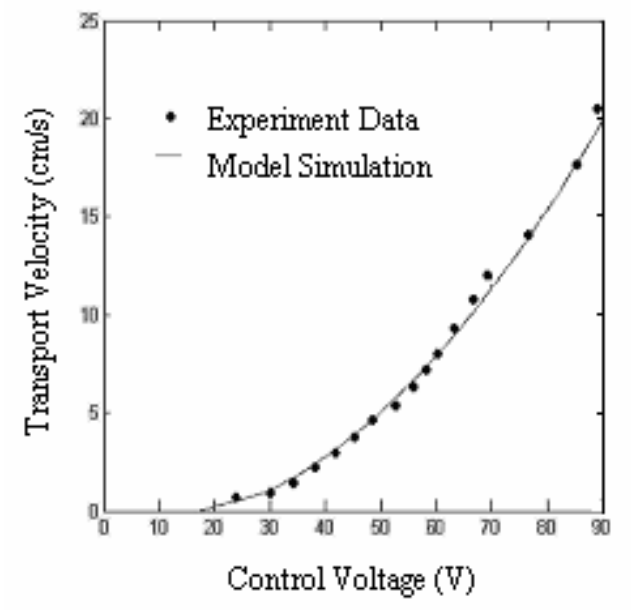

Figure 5. Variation of transport velocity with control voltage.

\subsection{Online Testing of Catastrophic Faults}

Most catastrophic faults cause a complete cessation of droplet transportation at the system level. Therefore, we can easily detect these faults by using the testing scheme outlined in Figure 4. The fault site in this two-dimensional array is highlighted. Droplets are first driven along one direction, e.g. along the $\mathrm{x}$-axis, and they are observed at the other end of the array. Each row of the array transports a single droplet of fluid. Due to the catastrophic fault in Row 3, no droplet is observed for this row. As a result, the cells in this row are deemed as candidate faulty cells. Next, droplets are driven along the y-axis, and due to the fault in the array, no droplet is observed at the other end of Column 3. Thus we conclude that Column 3 contains a faulty cell. From the information about the faulty row and column, we can uniquely identify the faulty cell in the array.

The above illustration assumes that a catastrophic fault affects only one cell of the array. The testing technique described here can however be extended for locating multiple faulty cells, e.g., through the use of multi-step adaptive fault location methods. An important advantage of this approach is that it can be integrated into the droplet-manipulation-based microfluidic steps underlying a biomedical reaction, e.g. polymer chain reaction [20]. Concurrent testing can be carried out simultaneously with a biochemical reaction utilizing unused cells in the array, and a degree of fault tolerance can be achieved by reconfiguring the array such that the droplets avoid faulty cells in their flow paths.

\section{Parametric Fault Testing}

While catastrophic faults have the highest priority for detection as they result in complete malfunction, parametric faults are much harder to detect and may result in malfunction depending on the application domain and
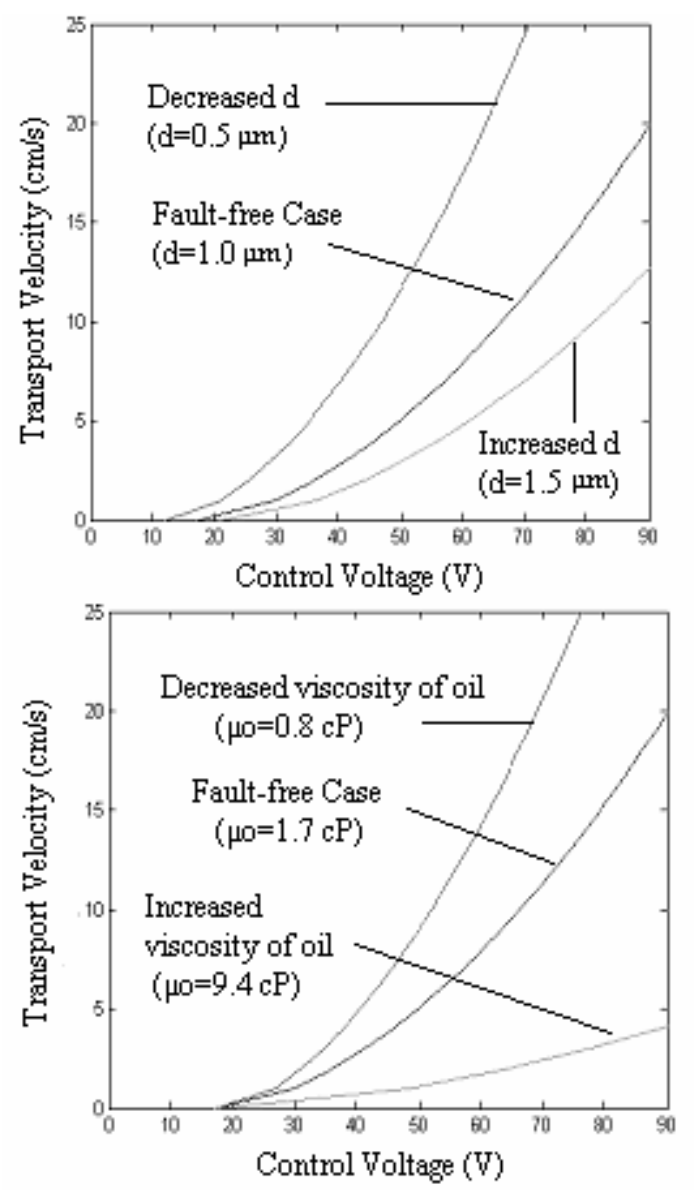

Figure 6. Effect of parametric variations on droplet velocity.

specifications. As a result, a viable concurrent testing scheme needs to consider both catastrophic and parametric faults. A large set of parametric faults that can arise from environmental effects, or from the degradation of system materials and may eventually lead to system failure if left undetected.

The degradation of the insulator thickness, and changes in the viscosity of the fluids are examples of such parametric faults that do not necessarily result in imminent loss of droplet movement, but rather result in degraded performance. For example, an increase in the viscosity of the filler fluid might impede the droplet motion, resulting in undesired fluid concentrations at the mixers. The identification of such deviations is essential during in-field operation in droplet-based microelectrofluidic systems. The analysis of fault manifestations requires an understanding of the fault-free behavior and the variations in this behavior with respect to the variations in underlying parameters.

\subsection{Fault-Free Model}

The behavioral model of droplet-based MEFS is based on the electrowetting actuation principle. We assume that 
the liquid flow within the droplet can be approximated as laminar flow, which requires that the Reynolds number of the droplet fluid be less than 1000. This assumption is valid because the Reynolds number of a nanoliter of fluid is usually no larger than 100 . The principle underlying this model is to balance the work done by the surface tension gradient force with the power dissipation. The power dissipation during the droplet transportation is caused by three factors: the viscosity and the resistance of the filler medium, the friction around the droplet/insulator surface and the internal viscous flow of the droplet. The following analytical model for droplet motion is derived in [23]:

$$
\frac{\varepsilon_{0} \varepsilon_{R}}{2 d} V^{2}-F_{T}=B\left(\frac{\mu_{d} U}{\gamma_{L M}}\right)^{0.3} \gamma_{L M}+\left(\frac{m L}{h}+s\right) \mu_{0} U+\zeta U
$$

where $V$ is the control voltage, $U$ is the transport velocity, $\varepsilon_{0}$ is permittivity of free-space, $\gamma_{L M}$ is the liquid-medium interfacial tension constant, and the remaining parameters and coefficients are as described in Tables 1 and 2 . Equation (1), covering all the significant physical phenomena in electrowetting, shows that the transport velocity of the droplet is a function of the control voltage and a number of relevant physical and fluidic parameters. We experimentally verified using laboratory apparatus that the analytical model accurately describes physical behavior. Figure 5 shows that the experimental data obtained from video frame counting matches simulation data for a wide range of values for the control voltage.

\begin{tabular}{|l|l|l|}
\hline $\begin{array}{l}\text { Parameter } \\
\text { name }\end{array}$ & Parameter description & $\begin{array}{l}\text { Nominal } \\
\text { value }\end{array}$ \\
\hline$d \quad(\mu \mathrm{m})$ & Thickness of dielectric layer & $10^{-4}$ \\
\hline$L \quad(\mu \mathrm{m})$ & Electrode length & 150 \\
\hline$h \quad(\mu \mathrm{m})$ & $\begin{array}{l}\text { Height between two parallel } \\
\text { plates }\end{array}$ & 88 \\
\hline$\mu_{0}(\mathrm{cP})$ & Viscosity of filler fluid & 1.7 \\
\hline$\mu_{d}(\mathrm{cP})$ & Viscosity of droplet & 1.9 \\
\hline
\end{tabular}

Table 1. Physical parameters affecting the droplet velocity.

\begin{tabular}{|l|l|l|}
\hline $\begin{array}{l}\text { Coefficient } \\
\text { name }\end{array}$ & Coefficient description & Value \\
\hline$F_{T}($ dyne $/ \mathrm{cm})$ & Threshold & 2.47 \\
\hline$B$ & Droplet viscous effect & 0.55 \\
\hline$m, s$ & Oil viscous effect & 28,112 \\
\hline$\zeta\left(\right.$ dyne s/cm $\left.{ }^{2}\right)$ & Contact-line friction & 0.4 \\
\hline$\varepsilon_{R}$ & $\begin{array}{l}\text { Relative permittivity of } \\
\text { insulator }\end{array}$ & 1.93 \\
\hline
\end{tabular}

Table 2. Numerical coefficients affecting the droplet velocity.

This behavioral model implies that the velocity of the droplet is a function of the environmental parameters, provided that the voltage is kept at a constant value. Thus, a defect in any of these parameters results in a deviation of the droplet velocity from the nominal value predicted by equation (1); see Figure 6.

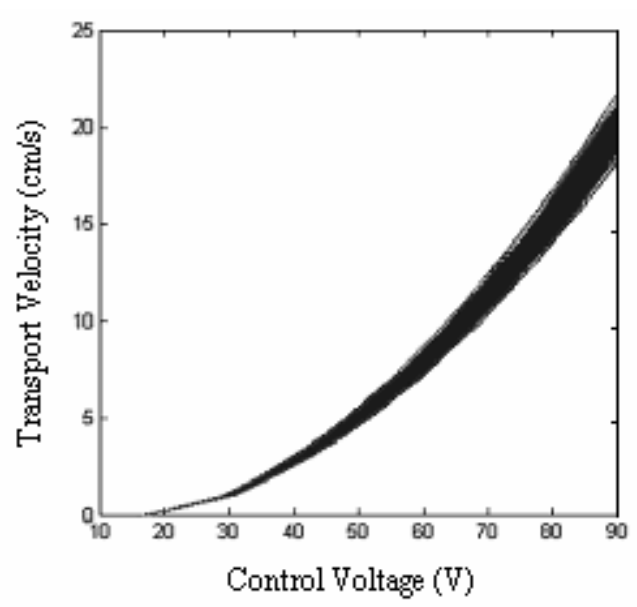

Figure 7. Range of acceptable values for droplet velocity.

Ideally, at a certain applied voltage, the droplet velocity is precisely determined by equation (1). However, in practice, all the parameters involved in equation (1) vary with a certain tolerance, resulting in a range of acceptable values for the droplet velocity, as illustraed in Figure 7. A viable parametric testing scheme has to take these variations into account to minimize the likelihood of a false alarm. Such parametric variations dictate certain upper and lower bounds on the droplet velocity in a faultfree system. These bounds can be computed through tolerance analysis [24].

Since parametric faults manifest as deviations in droplet velocity, parametric testing mainly consists of ensuring that the droplet velocity is within its bounds. Direct measurement of droplet velocity requires experimental methods such as video frame counting and capacitive sensing. All these methods, however, require external devices such as CCD cameras or oscillator circuits for capacitance measurement, which add unacceptable overhead and make testing cumbersome, especially in concurrent scenarios.

Fortunately, we can determine whether the droplet velocity is within the pre-determined bounds through a non-intrusive and cost-effective test set-up, which is based on the readouts of MEFS ports, and on adjustments in the duration of each electrode pulse.

\subsection{Lower-Bound Testing}

A parametric fault, such as an increase in the viscosity of the filler fluid, may slow the droplet beyond the acceptable value $U_{\min }$. In order to detect such effects, the droplet can be moved between two ports, a source and a sink, such that it can reach the sink only if its velocity is higher than the required minimum. Consider the droplet movement shown in Figure 8. For the droplet to move from the source to the sink, each electrode actuation has to be in the form of a pulse and the duration of the pulse has 


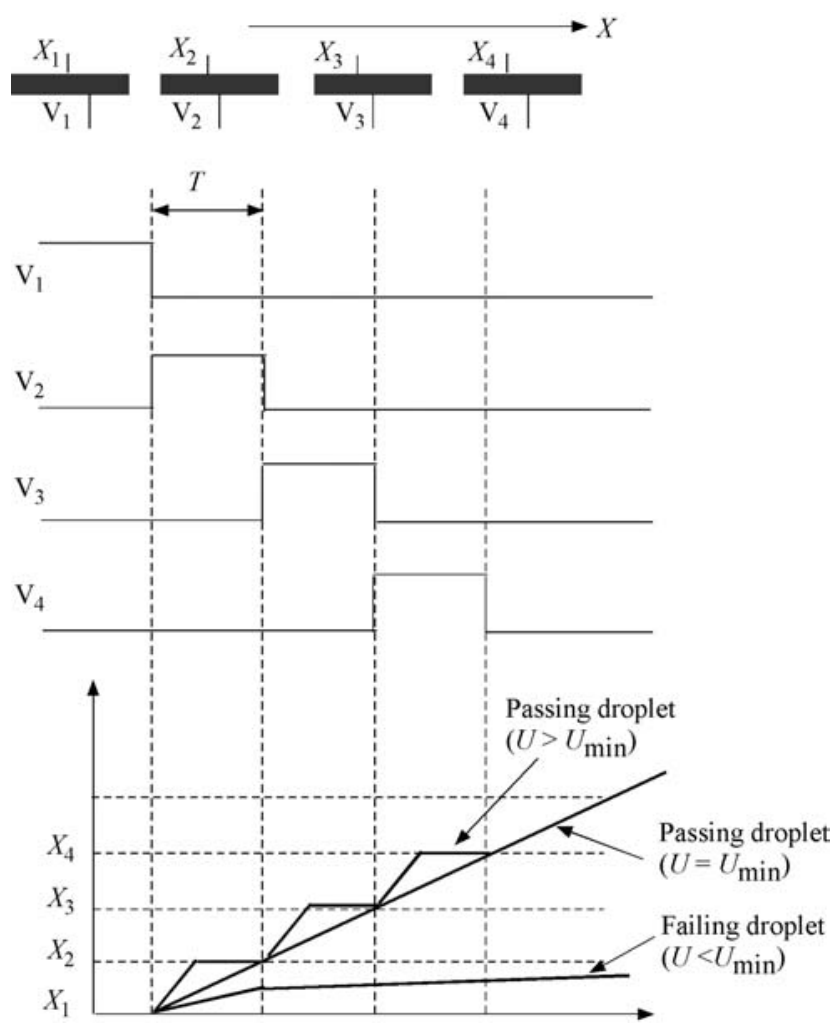

Figure 8. Source-to-sink droplet motion for lowerbound testing.

to be long enough for the droplet to reach the boundaries of the adjacant electrode. This is the boundary condition (corresponding to $U=U_{\min }$ ) under which the droplet can move continuously. If the actual velocity is larger than $U_{\min }$, the droplet will rapidly tranverse through the adjacent electrodes, and wait there until the next switching of control signal. On the other hand, if the velocity is less than $U_{\min }$, the droplet cannot keep up with the switching rate of the control electrodes. This implies that when the droplet reaches one electrode, the adjacent electrode has already been deactivated. For example, in Figure 8, if the voltage of $\mathrm{V}_{3}$ becomes zero before the droplet reaches $\mathrm{X}_{2}$, the droplet will be stuck at some point between $X_{1}$ and $X_{2}$. As a result, the shortest pulse duration, $T$, that allows the droplet with the minimum acceptable velocity, $U_{\min }$, to reach its destination is given by: $T=L / U_{\min }$, where $L$ is the electrode length.

If the pulse duration is chosen appropriately, the droplets with velocities higher than $U_{\min }$ will reach the sink, and the droplets with lower velocities will fail to complete the movement, as illustrated in Figure 8.

\subsection{Upper-Bound Testing}

Upper-bound testing differs from lower-bound testing in the sense that the fault-free case is represented by the failure of a droplet to catch-up with the pulse frequency. However, testing for the upper-bound is more complicated

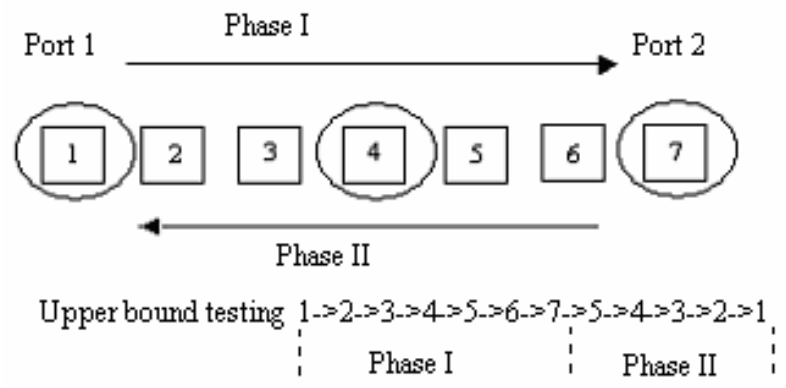

Figure 9. Two phase upper testing using bi-directional droplet motion.

than simply checking whether the droplet fails to reach the target since detecting the droplet's exit requires additional time, which violates the assumptions underlying the test scheme.

Fortunately, by slightly modifying the lower-bound testing scheme, we can test for the upper-bound of the droplet velocity, as illustrated in Figure 9. In this case, bidirectional droplet movement is utilized, where the source and the sink are the same port. In the first phase of the test, pulses of duration $T=L / U_{\max }$ are utilized, where $U_{\max }$ is the pre-computed upper bound on the droplet velocity. A droplet with an acceptable velocity $\left(U<U_{\max }\right)$ will not be able to reach the final electrode; it will be stuck at an intermediate position. In the second phase of the test, which begins right after the final electrode is deactivated, the droplet motion is set in the reverse direction. However, one electrode is skipped so as to keep the droplets that had reached the final electrode from moving. In this phase, the pulse duration is much higher to allow for the slower droplets to continue their motion. This backward pulsation will pick up the droplets that may have been stuck at intermediate points and move them towards the sink. Hence, the detection of a droplet at the end of the operation indicates a droplet velocity that is lower than the allowed maximum, thus a fault-free operation.

It is evident that this testing scheme can be combined with catastrophic fault testing to form a unified complete test methodology. It only needs to detect the ports of the MEFS without additional testing devices; this feature dramatically reduce the test cost. In addition, its simplicity facilitates concurrent testing.

\subsection{Evaluation of the Parametric Test Strategy}

The parametric testing strategy described here relies on the fact that parametric deviations result in an unexpected deviation in the droplet velocity. Equation (1) guarantees that a high-enough deviation in each parameter will eventually result in the velocity bound being violated. Hence the minimum-detectable deviations for all the 


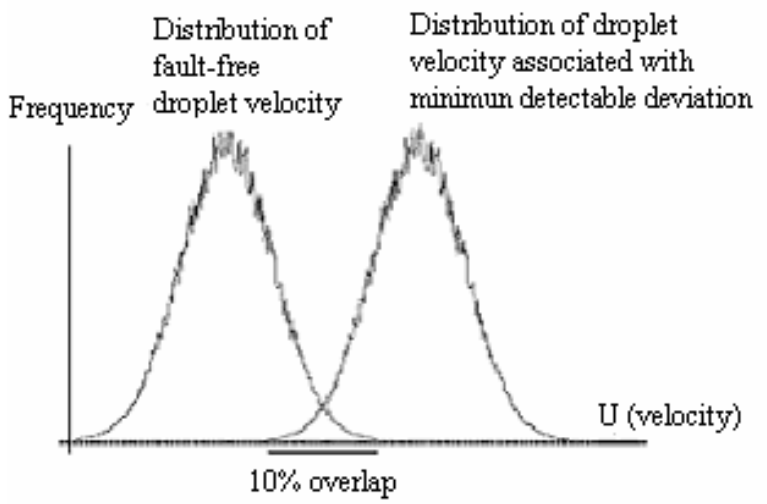

Figure 10. Detectable parameter deviations must result in a $10 \%$ or less overlap in velocity distributions.

parameters serve as a good evaluation metric in understanding the viability of the proposed testing scheme.

For a certain amount of deviation in a parameter to be detectable, it needs to shift the droplet velocity outside its determined bounds, while other parameters are allowed to vary within their tolerance. Since the droplet velocity for both the fault-free and faulty systems can be expressed in the terms of ranges, the detection of a certain parametric deviation can statistically be assured if droplet velocity ranges for the faulty and fault-free systems are nonoverlapping. However, since most physical parameters exhibit a Gaussian-like distribution, the non-overlapping criterion can be relaxed to include a $10 \%$ overlap, as illustrated in Figure 10, leading to a detection probability higher than $99.9 \%$.

\subsection{Experimental Set-Up}

In order to illustrate the viability of the parametric testing scheme, we have conducted simulation-based experiments and computed the velocity tolerance, as well as minimum-detectable deviation values for each physical parameter.

\subsubsection{Tolerance Analysis}

It is noted that the equation governing the relation between transport the velocity and the control voltage is nonlinear. The simplest and the most popular method for nonlinear statistical tolerance analysis is the Monte-Carlo simulation method [24]. Random values for each parameter are generated according to its distributions, and the value of the response function is computed for each set of parameter values. By generating very large samples, the tolerance response can be statistically analyzed.

In our case, it is assumed that all parameters, including geometrical parameters $(d, L, h)$ and fluidic parameters $\left(\mu_{0}\right.$, $\mu_{d}$ ), follow a Gaussian distribution with a mean value $\mu$ and a standard deviation $\sigma$.

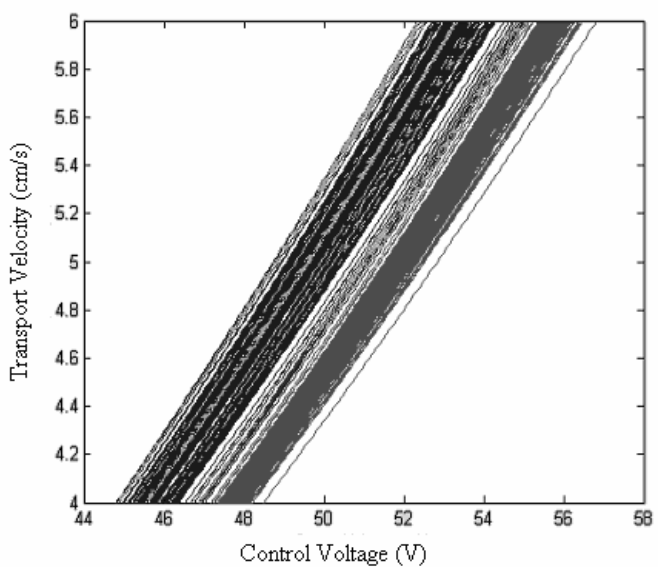

Figure 11. Results obtained with MonteCarlo simulations.

Using a 5\% tolerance for each physical and fluidic parameter, Monte-Carlo analysis has been carried out through MATLAB simulations (1000 runs). Figure 11 shows the results of the Monte-Carlo analysis over a range of control voltage values. For high control voltage values, the deviation in droplet velocity is higher as the sensitivity of parameter $d$ increases with the square of the control voltage, making this parameter the dominant variable. For a nominal control voltage value of $50 \mathrm{~V}$, the transport velocity tolerance is determined to be $7 \%$.

\subsubsection{Minimum Detectable Deviations}

In order to compute the minimum detectable deviations (MDDs) for each parameter, a linear search algorithm in conjunction with Monte-Carlo simulations is utilized, as illustrated in Figure 12. Even though linear search is quite inefficient, computational complexity is not an issue here due to the small number of parameters.

\begin{tabular}{|l|l|l|}
\hline Parameter & Nominal & MDD \\
\hline$d(\mu \mathrm{m})$ & $10^{-4}$ & $8.5 \times 10^{-6}(8.5 \%)$ \\
\hline$L(\mu \mathrm{m})$ & 150 & $91(60.7 \%)$ \\
\hline$H(\mu \mathrm{m})$ & 88 & $33(37.5 \%)$ \\
\hline$\mu_{0}(\mathrm{cP})$ & 1.7 & $0.28(16.5 \%)$ \\
\hline$\mu_{d}(\mathrm{cP})$ & 1.9 & $11(5.8 \times 100 \%)$ \\
\hline
\end{tabular}

Table 3. Minimum detectable deviations .

In order to determine the MDD for a parameter, $p$, we start with a deviation $\Delta p$ that exceeds the tolerance of $p$. Through Monte-Carlo simulations, the upper and lower bounds on the resulting transport velocity are determined. If the overlap between the fault-free and faulty ranges of the transport velocity is less than $10 \%$, the corresponding deviation, $\Delta p$, is considered to be detected. Since the actual distributions are Gaussion-like, such an overlap results in less than $0.1 \%$ probability of misclassification in terms of both false positives and false negatives. 


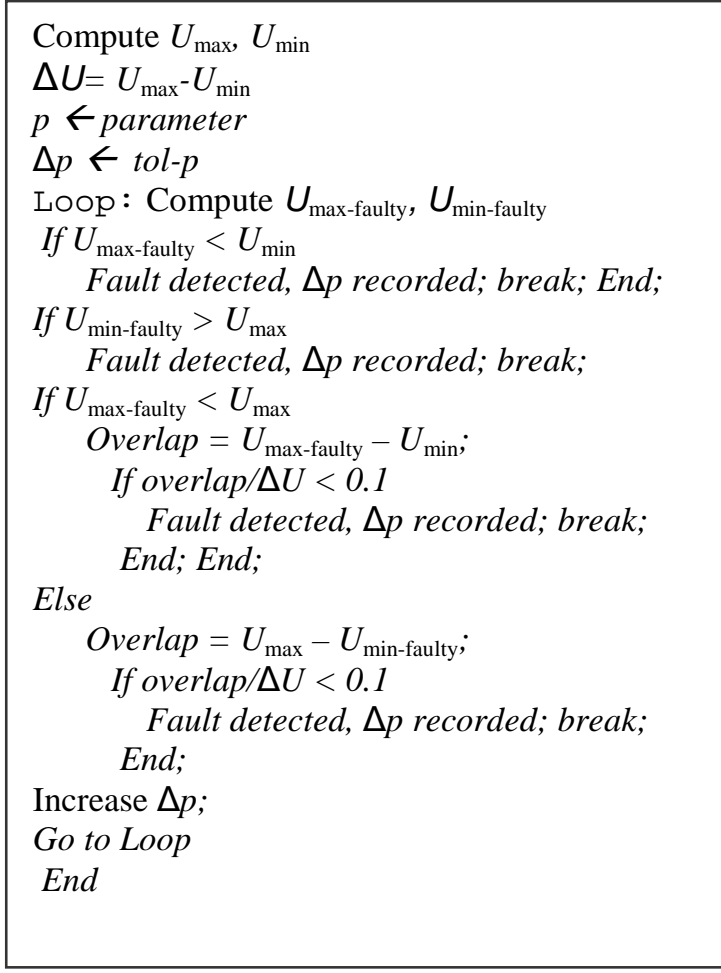

Figure 12. The linear search algorithm for computing the MDD of a parameter.

Table 3 shows the MDD values for the physical and fluidic parameters for a control voltage of $50 \mathrm{~V}$. For this particular set-up, the thickness of the dielectric layer $(d)$ has the most impact on the transport velocity. Thus, even small variations in $d$ can be detected using the proposed concurrent detection method. On the other extreme, the viscosity of the droplet, $\mu_{d}$, has the least impact on the transport velocity, leading to a high MDD value. It is worthwhile to note that the viscosity of the filler medium, $\mu_{0}$, and the viscosity of the droplet, $\mu_{d}$, are closely related, and are affected by the same environmental changes. As a result, a change in $\mu_{d}$ is associated with a similar change in $\mu_{0}$, and thus is detected at a much lower deviation than as suggested in Table 3. In addition, by using various control voltage values, the sensitivities of a number of parameters can be adjusted. This multi-phase testing scheme deceases the MDD values for all parameters involved.

\section{Conclusions}

In this paper, we have presented the first attempt to define a fault model and develop a test methodology for a droplet-based microelectrofluidic system (MEFS). As MEFS become widespread in safety-critical biomedical applications, dependability emerges as a critical performance parameter. We have developed a costeffective concurrent test methodology to increase the dependability of droplet-based MEFS. We have presented a classification of catastrophic and parametric faults in
MEFS and shown how faults can be detected by electrostatically controlling and tracking droplet motion. A tolerance analysis method based on Monte-Carlo simulation has been developed to characterize the impact of parameter (both physical and fluidic) variations on system performance. In our ongoing work, we are investigating the diagnosis of parametric faults that affect system performance.

\section{Acknowledgement}

The authors thank Dr. Tianhao Zhang of Cadence Design Systems, and Hong Ren of Duke University for their valuable comments. The work on droplet-based microelectrofluidic systems at Duke University has been pioneered by Prof. Richard Fair, Dr. Michael Pollack and Dr. Vamsee Pamula, and we thank them for sharing their insight with us.

\section{References}

[1] Z. Juneidi, K. Torki, S. Martinez, G. Nicolescu, B. Courtois and A. Jerraya, "Global modeling and simulation of system-on-chip embedding MEMS devices", Proc. International Conference on ASIC, pp. 666-669, 2001.

[2] S. Mir, H. Kerkhoff, R.D. Blanton, H. Bederr, H. Klim, "SoCs with MEMS? Can we include MEMS in the SoCs design and test flow?", Proc. IEEE VLSI Test Symposium, p. 449, 2002.

[3] T. Zhang, K. Chakrabarty and R. B. Fair, Microelectrofluidic Systems: Modeling and Simulation, CRC Press, Boca Raton, FL, 2002.

[4] W. Menz and A. Guber, "Microstructure technologies and their potential in medical applications", Minimally Invasive Neurosurgury, vol. 37, pp.21-27,1994.

[5] S. Shoji, "Microfabrication technologies and micro flow devices for chemical and bio-chemical micro flow systems", Proc. Microprocesses and Nanotechnology Conf., pp. 72-73, 1999.

[6] M. A. Burns et al., "An integrated nanoliter DNA analysis device”, Science, vol. 282, pp. 484-487, 1998.

[7] R. G. Lerch et al., “A programmable mixed-signal ASIC for data acquisition systems in medical implants", Proc. IEEE int. Conf. Soild-State Circuits, pp. 162-163, 1995.

[8] B. Davies, "Robotics in minimally invasive surgery", IEE Colloquium Through the Keyhole: Microengoneering in Minimally Invasive Surgery, pp. 5/1-5/2, 1995.

[9] M. Fahndrich, B. Hochwind and A. Zollner, "Fluid dynamics in micro dosing actuators", Proc. SoildState Sensors and Actuators Conf., vol.2, pp. 295- 
298, 1995.

[10] International Technology Roadmap for Semiconductor (ITRS), http://public.itrs.net/Files/2001ITRS/Test.pdf.

[11] T. Thorsen, S. J. Maerkl and S. R. Quake, "Microfluidic large-scale integration", Science, vol. 298, pp. 580-584, 2002.

[12] A. Kolpekwar and R. D. Blanton, "Development of a MEMS testing methodology", Proc. International Test Conference, pp. 923-93, 1997.

[13] N. Deb and R. D. Blanton, "Analysis of failure sources in surface-micromachined MEMS", Proc. International Test Conference, pp. 739-749, 2000.

[14] H. G. Kerkhoff, "Testing philosophy behind the micro analysis system", Proc. SPIE: Design, Test and Microfabrication of MEMS and MOEMS, vol. 3680, pp.78-83, 1999.

[15] H. G. Kerkhoff and H. P. A. Hendriks, "Fault modeling and fault simulation in mixed micro-fluidic microelectronic Systems", Journal of Electronic Testing: Theory and Applications, vol. 17, pp. 427437, 2001.

[16] H. G. Kerkhoff and M. Acar, "Testable design and testing of micro-electro-fluidic arrays", Proc. IEEE VLSI Test Symposium, pp. 403-409, 2003

[17] J. L. Jackel, S. Hackwood, J. J. Veslka and G. Beni, "Electrowetting switch for multimode optical fibers", Applied Optics, vol. 22, pp. 1765-1770, 1999.

[18] M.G. Pollack, R.B. Fair and A.D. Shenderov, "Electrowetting-based actuation of liquid droplets for microfluidic applications", Applied Physics Letters, vol. 77, 2000.

[19] R. Yates, C. Williams, C. Shearwood and P. Mellor, "A micromachined rotating yaw rate sensor", Proc. Micromachined Devices and Components II, SPIE Meeting, pp. 161-168, 1996.

[20] J. Ding, K. Chakrabarty and R. B. Fair, "Scheduling of microfluidic operations for reconfigurable twodimensional electrowetting arrays", IEEE Transactions on Computer-Aided Design of Integrated Circuits \& Systems, vol. 20, pp. 14631468, December 2001.

[21] A. Jee and F. J. Ferguson, "Carafe: An inductive fault analysis tool for CMOS VLSI circuits", Proc. IEEE VLSI Test Symposium, pp. 92-98, 1993.

[22] M. G. Pollack, "Electrowetting-Based Microactuation of Droplets for Digital Microfluidics", PhD thesis, Duke University. 2001.

[23] H. Ren, R. B. Fair, M. G. Pollack, and E. J. Shaughnessy, "Dynamics of electro-wetting droplet transport", Sensors and Actuators B, vol. 87, pp. 201-206, 2002.
[24] S. D Nigam and J. U Turner, "Review of statistical approaches to tolerance analysis", Computer-Aided Design, vol.27, pp. 6-25, 1995. 